and the lower esophageal sphincter. Am J Gastroenterol 1987; 82: 622-624.

7 Lien HC, Chang CS, Chen GH. Can percutaneous endoscopic jejunostomy prevent gastroesophageal reflux in patients with pre-existing esophagitis? Am J Gastroenterol 2000; 95: 3439-3443.
8 McClave SA, Lukan JK, Stefater JA, et al. Poor validity of residual volumes as a marker for risk of aspiration in critically ill patients. Crit Care Med 2005; 33: 324-330.

\title{
Eradication of Pseudomonas aeruginosa in cystic fibrosis
}

\section{To the Editors:}

We read with interest the recently published article by TACCETTI et al. [1] in the European Respiratory Journal and the accompanying editorial by JONES [2]. TACETTI et al. [1] report on their experience with early eradication therapy against Pseudomonas aeruginosa (PA) in cystic fibrosis (CF), concluding that this treatment exerts a number of beneficial effects and is also cost-effective. However, as stated in the editorial [2], questions pertaining to many details of eradication therapy, such as the choice and combination of antibiotics, dosages and modes of delivery, and, in particular, the optimal duration of treatment remain as yet unanswered. We recently published the preliminary outcome of another eradication regimen against PA that had been introduced into the clinical routine of our centre in Graz (Austria) in 1999 [3]. A comparison of these two studies, both in terms of methodology and outcome, may shed some additional light on the previous questions.

There are considerable major differences in the methodology of these two studies. TACCETTI et al. [1] tried to eradicate PA with inhaled colistin and oral ciprofloxacin administered for 3 weeks. They continued treatment for a total of 3 months when PA was not eradicated after these first 3 weeks. Our more extensive eradication regimen consists of i.v. anti-PA therapy for 3 weeks, followed by oral ciprofloxacin for 6 weeks and inhaled anti-PA therapy b.i.d. (colistin or gentamicin) for the following 2 yrs. TACCETTI et al. [1] also assessed the effect of their eradication regimen on the basis of three consecutive negative cultures from "respiratory secretions" and negative serum antibody titres against PA within a 6-month period after treatment, backed up with an assessment of PA genotypes in a subgroup of their patients. In contrast, the bacteriological outcome in our study was defined on the basis of bronchoalveolar lavage fluid findings, in combination with three subsequent sputum samples. In the opinion of TACCETTI et al. [1], their results indicate true eradication. Unfortunately, this cannot be taken as actually proven in the individual patient for at least two reasons. First, oropharyngeal cultures (we assume that these are meant by "respiratory secretions", at least when referring to younger children) might not be as representative for defining bacterial colonisation of a CF lung as bronchoalveolar lavage fluid cultures [4]. Secondly, as actually shown by one of the authors of this paper, the time for immune responses after the onset of chronic PA lung infection differs from patient to patient and from antigen to antigen with reported mean times of 6-15 months [5]. Consequently, it might be misleading to rely on serum anti-PA antibodies for exclusion of PA infection in the short term. These differences in methodology raise a basic caveat as to the following comparison of the outcome of both studies.

Eradication was supposedly achieved in 47 (81\%) out of 58 newly colonised patients in the study of TACCETTI et al. [1] carried out in Florence. Our treatment regimen resulted in the eradication of PA in $28(90 \%)$ out of 31 patients. To some degree, this comparable initial success rate might indicate that eradication of PA could be achieved with somewhat less elaborate antimicrobial regimens than the one used in our study.

The rates of recolonisation and the duration of the PA-free periods differ markedly between the two studies. In total, 24 (51\%) patients from the centre in Florence were recolonised one or more times during the observation period as opposed to only eight $(29 \%)$ patients in our centre. The median PA-free period in our study (33 months, range 1-73) was almost twice as long as the one observed by TACCETTI et al. [1] (18 months, range $4-80)$. We speculate that these differences are most probably due to the prolonged inhalative anti-PA therapy that routinely follows PA eradication in our centre. In a previous study (that was cited in the above editorial [2], but mistakenly assigned to the Vienna CF centre instead to our centre in Graz), it was demonstrated that, in $\mathrm{CF}$ patients with defined risk situations for acquisition of PA, long-term prophylaxis with inhaled gentamicin can effectively delay such an acquisition [6]. As any CF patient after PA eradication can be deemed to be at an increased risk of PA reacquisition, this situation may also call for long-term prophylactic inhalation of antibiotics. However, one could speculate that this strategy might foster the emergence of other and multiresistant microorganisms in the respiratory tract. Presently, we do not hold any data that further substantiate this concern, but, clearly, further close observation is warranted.

In conclusion, given the encouraging results of a number of cystic fibrosis centres with different Pseudomonas aeruginosa eradication protocols, placebo-controlled prospective studies appear to become ethically questionable. As a consequence, comparisons between studies employing different 
methodologies in different settings may be the only way to develop optimised eradication regimens.

\section{E. Eber, G.H. Thalhammer and M.S. Zach}

Respiratory and Allergic Disease Division, Paediatric Dept, Medical University of Graz, Graz, Austria.

\section{REFERENCES}

1 Taccetti G, Campana S, Festini F, Mascherini M, Döring G. Early eradication therapy against Pseudomonas aeruginosa in cystic fibrosis patients. Eur Respir J 2005; 26: 458-461.

2 Jones AM. Eradication therapy for early Pseudomonas aeruginosa infection in CF: many questions still unanswered. Eur Respir J 2005; 26: 373-375.

3 Thalhammer GH, Pratl B, Eber E, Zach MS. Pseudomonas aeruginosa in patients with cystic fibrosis: a comparison of early eradication and conventional therapy. Eur Respir $J$ 2004; 24: Suppl. 48, 385s-386s.

4 Armstrong DS, Grimwood K, Carlin JB, Carzino R, Olinsky A, Phelan PD. Bronchoalveolar lavage or oropharyngeal cultures to identify lower respiratory pathogens in infants with cystic fibrosis. Pediatr Pulmonol 1996; 21: 267-275.

5 Döring G, Høiby N. Longitudinal study of immune response to Pseudomonas aeruginosa antigens in cystic fibrosis. Infect Immun 1983; 42: 197-201.

6 Heinzl B, Eber E, Oberwaldner B, Haas G, Zach MS. Effects of inhaled gentamicin prophylaxis on acquisition of Pseudomonas aeruginosa in children with cystic fibrosis: a pilot study. Pediatr Pulmonol 2002; 33: 32-37.

DOI: $10.1183 / 09031936.06 .00118805$

\section{ERRATA}

“GUIDELINES FOR THE MANAGEMENT OF ADULT LOWER RESPIRATORY TRACT INFECTIONS". M. WOODHEAD, F. BLASI, S. EWIG, G. HUCHON, M. IEVEN, A. ORTQVIST, T. SCHABERG, A. TORRES, G. VAN DER HEIJDEN AND T.J.M. VERHEIJ. EUR RESPIR J 2005; 26: 1138-1180.

Unfortunately, one of the authors' names was printed incorrectly. M. Leven should read M. Ieven.

DOI: $10.1183 / 09031936.06 .50055705$

“EXPIRATORY FLOW LIMITATION AND OBSTRUCTION IN THE ELDERLY”. C. DE BISSCHOP, M.L. MARTY, J.F. TESSIER, P. BARBERGER-GATEAU, J.F. DARTIGUES AND H. GUÉNARD. EUR RESPIR J 2005 ; 26 : 594-601.

Unfortunately, figure 1 was presented incorrectly as shown and should have appeared as follows.
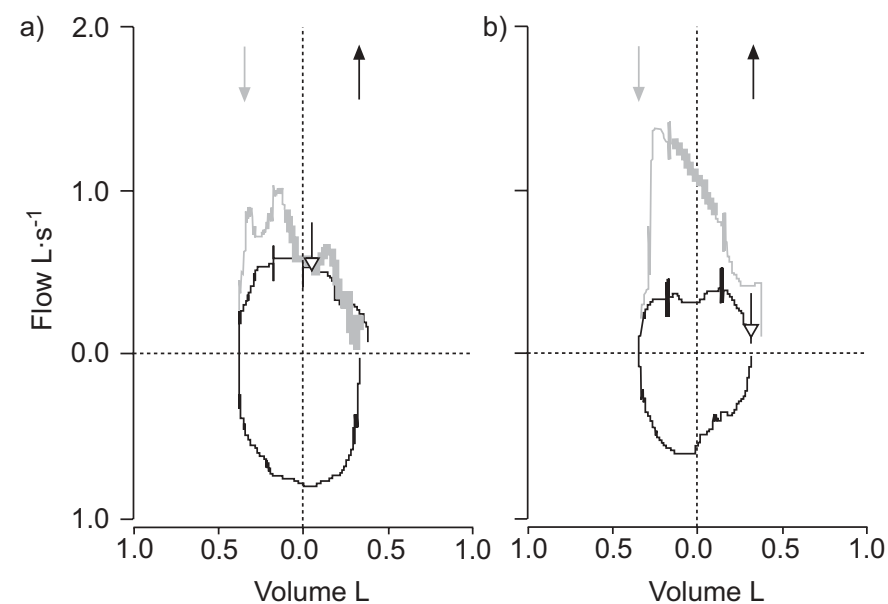

FIGURE 1. a) Flow/volume loops before and during a 5 hectopascal negative expiratory pressure technique (black trace) from an expiratory limited female with dyspnoea (grade 2) with no medical history, in the expiratory limitation and dyspnoea due to age group. The female was: aged $74 \mathrm{yrs}, 1.44 \mathrm{~m}$ in height and weighed $64 \mathrm{~kg}$. The subject had a forced expiratory volume (FEV1) of $1.21 \mathrm{~L}$, a forced vital capacity $(F V C)$ of $1.53 \mathrm{~L}$ and a $\mathrm{FEV}_{1} / \mathrm{FVC}$ ratio of $79.1 \%$. b) A healthy 71-yr-old female, with a height of $1.55 \mathrm{~m}$, a weight of $53 \mathrm{~kg}$, a FEV1 of $1.76 \mathrm{~L}$, a FVC of $2.25 \mathrm{~L}$, and a FEV $1 / F V C$ ratio of $78.5 \%$. The grey arrow denotes the beginning of the negative expiratory pressure technique (NEP) and the black arrows denote the end of the NEP. The open arrow denotes limitation. The grey traces represent the flow/volume curves while the subjects breathed with NEP.

For the Support statement, it should be noted that GlaxoSmithKline is situated in Marly le Roi, not in Bordeaux. 